\title{
Metodología de entrenamiento aeróbico utilizado por profesionales del fitness
}

\author{
Francys Paula Cantieri** \\ Mauro Virgílio Gomes de Barros ${ }^{* * *}$ \\ Gustavo Aires de Arruda*t* \\ Antonio Carlos Gomes ${ }^{* * * * * *}$ \\ Ágata Cristina Marques Aranha ${ }^{* * * * * *}$
}

Recibido: julio 5 de 2019 • Aceptado: septiembre 10 de 2019

\begin{abstract}
* Artículo de investigación. Este estudio fue financiado, en parte, por la Coordinación para la Mejora del Personal de Educación Superior (CAPES) y por la Fundación Estatal de Ciencia y Tecnología de Pernambuco (FACEPE). Universidad de Pernambuco, Recife. Brasil. Citar como: Cantieri, F., Gomes, M., Aires de Arruda, G., Gomes, A. y Aranha, A. (2019). Metodología de entrenamiento aeróbico utilizado por profesionales del fitness. Revista de Investigación Cuerpo, Cultura y Movimiento, 9(1), 77-100. DOI: https://doi.org/10.15332/2422474x/5354
\end{abstract}

"* Pós-Doutorado em Educação Física, pelo programa associado de pós graduação UPE/ ufPB, membro do Grupo de Pesquisa em Estilos de Vida e Saúde - GPEs, Bolsista do Programa Nacional de Pós Doutorado da Capes (PNPD) com incentivo da Fundação de Amparo à Ciência e Tecnologia do Estado de Pernambuco Recife, Pernambuco, Brasil. Correo electónico: francyspaulapersonal@gmail.com / ORCID: https://orcid.org/0000-0002-1132-4540

"*** Pós-Doutorado em Educação Física, profesor asociado, Universidade de Pernambuco, Grupo de Pesquisa em Estilo de Vida e Saúde - GPEs, Recife PE, Brasil. Correo electónico: mauro.barros@upe.br / ORCID: http://0000-0003-3165-0965

***** Pós-doutorado Educação Física, Universidade de Pernambuco, Recife PE, Brasil. Correo electónico: arrudaga@yahoo.com.br / oRCID: http://orcid.org/0000-0002-9157-6114

***** Pós-Doutorado em Educação Fìsica, Instituto Olímpico Brasileiro, São Paulo, Brasil. Correo electónico: contatoacgomes@gmail.com/ orCID: http://orcid.org/0000-0002-3001-8311

***** Pós-Doutorado em Ciências do Desporto, Universidade Tras-os-Montes e Alto Douro, Vila Real, Portugal. Correo electónico: aaranha@utad.pt / orcID: http://orcid. org/0000-0003-3593-0825 


\section{Resumen}

La capacidad aeróbica es un componente importante de la aptitud física relacionada con la salud; su planificación es fundamental para un programa de ejercicios. El objetivo de este estudio fue diagnosticar la metodología utilizada por los profesionales del fitness para prescribir ejercicios aeróbicos y su correspondencia con los valores propuestos en la literatura. Participaron 452 profesionales masculinos y femeninos de Londrina (Estado de Paraná) y São Paulo (Estado de São Paulo). Se utilizó un cuestionario que contenía 46 preguntas relacionadas con la metodología del ejercicio físico, en el que solo se consideraron las preguntas asociadas con la capacidad aeróbica. La mayoría de los profesionales prescribió entre el $61 \%$ y el $80 \%$ del $\mathrm{vo}_{2}$ máximo, adoptó la frecuencia cardíaca (FC) entre 141 y 160 latidos por minuto (ВРм), usó entre 31 y 45 minutos en el entrenamiento de pérdida de grasa corporal y utilizó la ergometría como un medio de práctica. La mayoría de ellos no realizaron pruebas de aptitud aeróbica en sus programas de ejercicio. En general, los profesionales utilizaron valores porcentuales de $\mathrm{VO}_{2}$ máximo, $\mathrm{FC}$ y duración de la estancia para la pérdida de grasa, correspondientes a los recomendados en la literatura.

Palabras clave: prescripción de ejercicio, metodología de ejercicio físico, capacidad aeróbica, práctica profesional. 


\section{Aerobic training methodology used by fitness professionals}

\section{Abstract}

Aerobic capacity is an important component of health-related fitness and planning it is essential for an exercise program. The objective of this study was to diagnose the methodology used by fitness professionals to prescribe aerobic exercises and their correspondence with the values proposed in the literature. Four hundred fifty-two (452) male and female professionals from Londrina (PR) and São Paulo (SP) participated. A questionnaire containing 46 questions related to the methodology of physical exercise was used, in which only questions related to aerobic capacity were considered. Most professionals prescribed between $61 \%$ and $80 \%$ of the $\mathrm{VO}_{2}$ max, adopted HR between 141 and 160 врм, used between 31 and 45 minutes in the training of body fat loss and used ergometry as a means of practice. Most of them did not perform aerobic fitness tests in their exercise programs. Overall, professionals used percentage values of $\mathrm{vO}_{2}$ max, HR and length of stay for fat loss, corresponding to those recommended in the literature.

Keywords: exercise prescription, physical exercise methodology, aerobic capacity, professional practice. 


\section{Introducción}

La inactividad física se reconoce como un factor de riesgo de enfermedad coronaria (Blair et al., 1989; Fletcher, Blair et al, 1992; Warren et al, 2010; Church, LaMonte, Barlow y Blair, 2005) y el ejercicio regular desempeña un papel preventivo, ya que aporta numerosos beneficios a las personas de diferentes grupos de edad y reduce las causas de mortalidad cuando participan en programas de ejercicio o incluso cambian su comportamiento y adoptan un estilo de vida más activo (Warren et al., 2010; Kavouras et al., 2007; Garber et al., 2011; Sui et al., 2007) .

Se debe diseñar un programa de ejercicio físico para satisfacer las necesidades de los profesionales en términos de indicadores de salud y estado físico. En este sentido, uno de los objetivos por alcanzar es la mejora de la aptitud cardiorrespiratoria (aeróbica/anaeróbica), a través de actividades que promuevan adaptaciones fisiológicas en el sistema de producción de energía, ya que los altos niveles de aptitud cardiorrespiratoria están asociados con bajos riesgos de enfermedades (Church et al., 2005; Garber et al., 2011).

Se recomiendan ejercicios de baja habilidad como caminar, andar en bicicleta y nadar para desarrollar la capacidad aeróbica, también llamados actividades de resistencia. El programa de entrenamiento de resistencia utiliza cargas progresivas como principio, lo que permite adaptaciones fisiológicas y mejoras en el estado de salud. Además, está relacionado con varios objetivos como la pérdida de peso, el rendimiento deportivo, la rehabilitación cardíaca, entre otros (Garber et al., 2011; Leitzmann et al., 2007). Su prescripción se basa en la manipulación de la intensidad, duración, frecuencia, progresión y modalidad practicada, y debe considerar algunos factores intervinientes como la edad, el sexo y la condición física general (Donnelly, et al., 2009).

La intensidad del entrenamiento está directamente relacionada con el gasto de energía; por lo tanto, a medida que aumenta, hay un mayor reclutamiento de unidades motoras de los músculos involucrados en la acción, aumentando así la capitación de oxígeno linealmente, hasta alcanzar su capacidad máxima. La evaluación de la intensidad del ejercicio se puede definir por el porcentaje de consumo máximo de oxígeno $\left(\% \mathrm{VO}_{2 \text { máx }}\right)$, porcentaje de frecuencia cardíaca máxima (\% HR), frecuencia cardíaca de reserva 
(\% HR reserva) y gasto de actividad metabólica (МЕT) (Garber et al., 2011; Gomes, Perez, Carletti y Marques, 2016).

De acuerdo con las recomendaciones del American College of Sports Medicine (ACSM), la intensidad apropiada para desarrollar la capacidad aeróbica en adultos debe ser entre $50 \%$ a $85 \%$ del $v_{2 \text { máx }}$ o $60 \%$ a $90 \%$ de la frecuencia cardíaca máxima prevista (Garber et al., 2011).

Por lo general, el $\mathrm{vO}_{2 \text { máx }}$ se ha utilizado como un indicador importante para la evaluación de la aptitud aeróbica, tanto en atletas como en individuos con baja actividad, y es considerado el índice que mejor representa la potencia aeróbica; es decir, la cantidad máxima de energía que puede producir el metabolismo aeróbico en un intervalo de tiempo dado (Caputo y Denadai, 2004; Amorin, 2002; Rosenberger, Meyer Gäßler, Faude y Kindermann, 2010; Silva, Franklin, Forman y Araújo, 2016; Araújo, Herdy y Stein, 2013). Idealmente, se mide directamente a través del análisis de gases exhalados durante una prueba de ejercicio, o indirectamente a través de ecuaciones predictivas que, incluso con errores entre $15 \%$ y $20 \%$, son adoptadas por los investigadores debido a su fácil aplicabilidad y bajo costo (Amorin, 2002; Araújo, Herdy y Stein, 2013).

Según Denadai (1995), varios factores pueden influir en el $\mathrm{vO}_{2 \text { máx }}$, incluidos la genética, el sexo, la edad, el nivel de condición física, la capacidad de ventilación pulmonar, la difusión alveolocapilar de oxígeno, el sistema de transporte y la diferencia arteriovenosa. Mantener el $\mathrm{vo}_{2 \text { máx }}$ a niveles saludables puede reducir el riesgo de mortalidad; según Myers, Prakash, Froelicher, Do, Partington y Atwood (2002) un aumento de $3.5 \mathrm{~mL}$ en el oxígeno corresponde a un aumento del $12 \%$ en la esperanza de vida del individuo.

La dificultad en la medición directa del $\mathrm{vO}_{2 \text { máx }}$ y el porcentaje de posibles errores en las mediciones indirectas hace que la frecuencia cardíaca sea ampliamente utilizada para la prescripción y el control del ejercicio físico. Este se basa en zonas de entrenamiento relacionadas con la vía metabólica de la energía, o se determina a través de medidas respiratorias y respiratorias (Zakarohv y Gomes, 2003; Seiler y Kjerland, 2006; Gilman, 1996). Su modulación se produce en una acción conjunta de las ramas simpática y parasimpática del sistema nervioso autónomo, y sus respuestas y adaptaciones son objeto de investigación científica, desde la más simple hasta la más sofisticada; incluso se señala como la información más destacada extraída 
de una prueba de ejercicio cardiopulmonar máximo (Raxwal, et al., 2001; Almeida, 2007).

A pesar de sus limitaciones, el uso de la FC en el control y la prescripción del entrenamiento aeróbico, especialmente en individuos poco entrenados o en programas de pérdida de peso, aún proporciona buenos resultados, siempre que se investigue su comportamiento en relación con diferentes situaciones para su correcta aplicación (Carvalho y Campbell, 2012). Incluso, hoy en día las dificultades para realizar pruebas directas que determinen $\mathrm{el} \mathrm{vO}_{2 \text { máx }}$ lideran a los profesionales que prescriben ejercicios aeróbicos, para adoptar tablas que contengan zonas de entrenamiento con valores absolutos de frecuencia cardíaca objetivo (Gilman, 1996; Garber et al., 2011; Amorin, 2002; Foster, Florhaug, Franklin, Gottschall, Hrovatin, Parker y Dodge, 2001).

Considerando la información sugerida por Zahakov y Gomes (2003), donde definen cinco zonas de entrenamiento y sus respectivos valores de la FC (Figura 1) relacionados con el porcentaje máximo de vo2 para cada una de estas zonas, se observa que los valores de porcentaje de $\mathrm{vo}_{2}$ - los valores máximos indicados en la literatura para el entrenamiento de capacidad aeróbica ( $50 \%$ a $85 \%$ ) - se refieren a las zonas aeróbicas (hasta 140 врм) у al umbral aeróbico (140 a 160 в PM). Esta relación se utiliza comúnmente en la práctica por profesionales que prescriben ejercicios aeróbicos; por lo tanto, estos valores se adoptarán para analizar los resultados de este estudio.

Tabla 1. Zonas de frecuencia cardíaca

\begin{tabular}{ccc}
\hline n. $^{\circ}$ & Zonas & FC (por min) \\
\hline I & Aeróbica & Hasta 140 \\
II & Aeróbica (umbral) & 140 a 160 \\
III & $\begin{array}{c}\text { Mezclado } \\
\text { (aeróbica-anaeróbica) } \\
\text { IV }\end{array}$ & Anaeróbica glucolítico \\
V & Anaeróbico (aláctico) & Arriba de 180 \\
\hline
\end{tabular}

Fuente: adaptado de Zakharov y Gomes (2003). Ciencia do treinamento desportivo. 
Los estudios clásicos revelaron una linealidad entre la intensidad del esfuerzo y la magnitud de la FC. En los ejercicios continuos con intensidad constante la demanda de energía no cambió, por lo que no cambió el suministro de oxígeno a los músculos. En contraste, en situaciones donde hubo un progreso en la intensidad, aumentaba la magnitud de la FC (Astrand y Rodahl, 1986). Otras investigaciones refutan esta relación y muestran en sus resultados con individuos sometidos a esfuerzos progresivos una gran variabilidad de los recursos humanos, lo que provoca posibles malas interpretaciones.

Lo que sería probable en el esfuerzo máximo durante un ejercicio, debido a una mayor estimulación simpática y a una menor estimulación parasimpática, sería el mantenimiento de la FC constante; es decir, sin variabilidad. Sin embargo, los estudios mostraron que durante la prueba de esfuerzo máximo la FC permaneció variable con el tiempo, lo que sugiere que los mecanismos de control de la FC en el ejercicio máximo no parecen ser exclusivamente autónomos (Amorin, 2002; Almeida, Ricardo y Araújo, 2005; Casties, Mottet y Le Gallais, 2006; Skinner, Gaskill, Rankinen, Leon, Rao, Wilmore y Bouchard, 2003).

Además, según Kenney, Wilmore y Costill (2013), el uso de la frecuencia cardíaca como receta es eficiente, porque, incluso en situaciones o entornos adversos (calor, altitud, etc.), si el individuo intenta mantener el trabajo a niveles normales (velocidad de carrera) la FC sería más alta; en estas situaciones el profesional cambiaría la intensidad de su trabajo para cumplir con la FC prescrita.

En el estudio clásico de Karvonen, que definió un porcentaje de intensidad alrededor del $70 \%$ de la FC de reserva - obtenida por la diferencia entre la FC máxima (definida por la fórmula 220 - edad) y la FC en reposocomo ideal para desarrollar la capacidad reserva, se afirma que el porcentaje de reserva de la FC se ha utilizado rutinariamente como una medida alternativa al $\mathrm{vo}_{2 \text { máx }}$ para la prescripción de ejercicio, y su uso se basa en la relación lineal entre esta variable y el $\mathrm{vO}_{2 \text { máx }}$, lo cual es ampliamente descrito en la literatura científica (Arts y Kuipers, 1994; Cunha, Midgley, Monteiro y Farinatti, 2010; Swain y Franklin, 2002; Swain, Leutholtz, King, Haas y Branch, 1998).

Contrario a estas afirmaciones, otros estudios no han revelado una equivalencia entre estas variables en las diferentes poblaciones evaluadas (Swain y Leutholtz, 1997; Swain et al., 1998; Panton, Graves, Pollock, Garzarella, 
Carroll, Leggett, Lowenthal y Guillen, 1996; Weltman, Weltman, Rutt, Seip, Levine, Snead, Kaiser y Rogol, 1989). Swain y Leutholtz (1997), basados en sus resultados, propusieron un concepto de reserva de $\mathrm{vO}_{2}$ obtenido por la diferencia entre el $\mathrm{VO}_{2 \text { máx }}$ y el $\mathrm{vO}_{2}$ en reposo, y señalaron una equivalencia entre el porcentaje de reserva de $\mathrm{vO}_{2}$ y el porcentaje de reserva de la $\mathrm{FC}$ en adultos sanos. A partir de entonces, el ACSM comenzó a recomendar el porcentaje de reserva de la $\mathrm{FC}$ o el porcentaje de reserva del $\mathrm{vO}_{2}$ para la prescripción de ejercicio, lo que sugiere intensidades entre $20 \%$ y $85 \%$.

Estudios destinados a comparar las implicaciones prácticas y fisiológicas de las diferentes prescripciones de ejercicio basadas en el $\mathrm{vo}_{2 \text { máx }} \mathrm{y}$ de reserva, y del HR máximo y de reserva, sugieren que no hay evidencia de superioridades en el $\mathrm{VO}_{2}$ de reserva como indicador efectivo para promover cambios fisiológicos en el profesional (Gormley, Swain, High, Spina, Dowling, Kotipalli y Gandrakota, 2008; Mann, Lamberts y Lambert, 2013; Lounana, Campion, Noakes y Medelli, 2007; Garber et al., 2011). Con base en estas notas, la última publicación del ACSM ha incluido nuevamente valores de intensidad basados en el $\mathrm{vO}_{2 \text { máx }}$ y la FC máxima en sus pautas. Ya sea a través de la FC o del $\mathrm{vO}_{2 \text { máx }}$, la dosificación del ejercicio cardiorrespiratorio es una función de la intensidad y el volumen del entrenamiento, que junto con el tipo de práctica constituyen los componentes básicos de su prescripción.

Actualmente, las pautas para prescribir la capacidad aeróbica en la literatura recomiendan al menos 150 minutos de actividades por semana, realizadas de 3 a 5 veces por semana, con intensidades moderadas que van del $40 \%$ al $65 \%$ de vo $_{2 \text { máx }}$ o del $55 \%$ al $76 \%$ de la FC máxima; o 75 minutos por semana, con intensidades vigorosas que van del $64 \%$ al $90 \%$ del $\mathrm{vO}_{2 \text { máx }}$ o del $70 \%$ al $95 \%$ de la FC (Organización Mundial de la Salud [oms], 2010; Garber et al., 2011; Mendes, Sousa y Barata, 2011).

Las recomendaciones de Donnelly y colaboradores (2009) con respecto a la prescripción de entrenamiento para la pérdida de peso corporal, tienen un tiempo de práctica de al menos 250 a 300 minutos por semana, con frecuencias entre 3 y 5 veces por semana, con intensidad moderada (entre $40 \%$ y $65 \%$ del $\operatorname{vo}_{2 \text { máx }}$ o $55 \%$ a $76 \%$ de la FC máxima). También señalan que un período más largo de práctica o intensidad afecta positivamente el gasto de energía y, en consecuencia, la pérdida de peso. 
A pesar de la existencia de un marco metodológico bien descrito en la literatura, el uso de esta información en la práctica por parte de los profesionales que trabajan con ejercicio físico aún no está consolidado. Además, la integración entre la evidencia científica y la metodología utilizada por los profesionales de educación física merece ser estudiada con la intención comprender si la prescripción de ejercicios físicos propuesta por los profesionales está orientada con base en el conocimiento académico o, por el contrario, si difiere de lo que revela la ciencia (Verenguer, 2003).

En este sentido, surgió la siguiente pregunta de estudio: ¿je consideran las bases teóricas del entrenamiento cardiorrespiratorio descritas en la literatura en las estrategias metodológicas de los profesionales que trabajan con la prescripción de ejercicios físicos? El objetivo de este estudio fue diagnosticar la metodología utilizada en la prescripción de ejercicios cardiorrespiratorios por profesionales que trabajan con ejercicios físicos y verificar si existe una correspondencia entre los valores prescritos por ellos y los recomendados en la literatura.

\section{Metodología}

El presente corresponde a un estudio de encuesta descriptivo. La muestra consistió en profesionales que trabajan con la práctica de ejercicios físicos y que tienen un registro profesional con el Consejo Federal de Educación Física (Confef). La colección se llevó a cabo entre estudiantes de programas de posgrado en las ciudades de Londrina (PR) y São Paulo (SP), y también en cursos y reuniones celebradas en el área en la ciudad de Londrina y la región.

El número total de participantes que completaron el cuestionario fue de 476 individuos, pero solo se incluyeron en los análisis a aquellos que respondieron todas las preguntas (452 individuos). Este estudio fue aprobado por el Comité de Ética en Investigación Humana del Centro de la Universidad de Filadelfia (Unifil) con el número 1013727.

El estudio incluyó a 452 profesionales de educación física de ambos sexos, debidamente registrados en el Consejo Federal de Educación Física. De los 314 hombres participantes, la gran mayoría afirmó desempeñarse como entrenador personal, con experiencia profesional de $6.5 \pm 5.6$ años; y la mayoría de ellos indicó realizar ejercicio físico en promedio 4 veces a 
la semana. Solo el $5.1 \%$ informó la presencia de algún tipo de enfermedad crónica y el $44.9 \%$ de ellos declararon tener ingresos mensuales entre 4 a 6 salarios mínimos.

Entre las 138 mujeres entrevistadas, $71.5 \%$ indicó trabajar como entrenadora personal, con experiencia profesional de $6 \pm 5.9$ años. Entre los profesionales, el $93.4 \%$ afirmó practicar ejercicio físico en promedio 4 veces a la semana. Aproximadamente, el $10 \%$ manifestó tener algún tipo de enfermedad crónica y casi el $50 \%$ de ellas tenían ingresos salariales más bajos que los hombres, entre 1 y 3 salarios mínimos (tabla 2).

\section{Instrumentos}

Como en la literatura no se encontró ningún instrumento que cumpliera con los objetivos de este estudio, se elaboró un cuestionario de 46 preguntas cerradas de opción múltiple y se agrupó en dos dimensiones: la primera titulada Metodología de entrenamiento (34 preguntas) y la segunda Marco de entrenamiento (12 preguntas). Este cuestionario cubrió aspectos relacionados con el método de entrenamiento, así como el desarrollo de habilidades motoras y variables de volumen e intensidad del ejercicio. Los valores de reproducibilidad de este instrumento fueron superiores a 0.7 , lo que permitió su uso. En este estudio las preguntas utilizadas fueron las relacionadas con el entrenamiento de la capacidad aeróbica.

\section{Procedimientos}

Después de aceptar participar en el estudio firmando el formulario de consentimiento libre e informado, el entrevistador ordenó a los sujetos que cumplían con los criterios de inclusión del estudio que respondieran todas las preguntas, indicando solo una de las alternativas y, en caso de duda, podría elegir la alternativa "Prefiero no responder". A los entrevistados no se les permitió indagar sobre la interpretación de las preguntas, evitando así la inducción en las respuestas. 
Tabla 2. Valores medios y de desviación estándar del perfil físico y el rendimiento de los profesionales de educación física que trabajan en la práctica del fitness

\begin{tabular}{|c|c|c|}
\hline Indicadores & Hombres $(\mathrm{n}=314)$ & Mujeres $(n=138)$ \\
\hline Edad (años) & $3.4 \pm 6.9$ & $31.1 \pm 6.5$ \\
\hline Masa corporal (kg) & $82.2 \pm 12.4$ & $63 \pm 9.8$ \\
\hline Estatura $(\mathrm{cm})$ & $176.4 \pm 9.22$ & $164.6 \pm 6.86$ \\
\hline $\operatorname{IMC}\left(\mathrm{KG} / \mathrm{M}^{2}\right)$ & $38.3 \pm 5.37$ & $43.2 \pm 6.1$ \\
\hline $\begin{array}{l}\text { Práctica profesional } \\
\text { (años) }\end{array}$ & $6.5 \pm 5.6$ & $5.9 \pm 5.9$ \\
\hline $\begin{array}{l}\text { Frecuencia semanal } \\
\text { de ejercicio }\end{array}$ & $4.53 \pm 1.3$ & $4.2 \pm 1.4$ \\
\hline \multicolumn{3}{|c|}{ ÁREA PROFESIONAL } \\
\hline Gimnasia & $14.7 \%$ & $16.7 \%$ \\
\hline Culturismo & $6.4 \%$ & $11.8 \%$ \\
\hline $\begin{array}{l}\text { Entrenador } \\
\text { personal }\end{array}$ & $78.9 \%$ & $71.5 \%$ \\
\hline \multicolumn{3}{|c|}{ INGRESO MENSUAL } \\
\hline 1 a 3 salarios & $35.1 \%$ & $49.2 \%$ \\
\hline 4 a 6 salarios & $44.9 \%$ & $40.2 \%$ \\
\hline Más de 7 salarios & $20 \%$ & $10.6 \%$ \\
\hline \multicolumn{3}{|c|}{ ENFERMEDADES CRÓNICAS } \\
\hline Ausencia & $94.9 \%$ & $90.4 \%$ \\
\hline Presencia & $5.1 \%$ & $9.6 \%$ \\
\hline \multicolumn{3}{|c|}{ EJERCICIO FÍsICO } \\
\hline Practicante & $93 \%$ & $93.4 \%$ \\
\hline No practicante & $6.8 \%$ & $6.6 \%$ \\
\hline
\end{tabular}

Fuente: elaboración propia. 
Después de completar el cuestionario, se le pidió a cada participante que completara un formulario de registro, que contenía información personal relacionada con la edad, mediciones antropométricas (masa corporal y altura), duración de la práctica profesional, actividad física, presencia de enfermedades crónicas, entre otros.

\section{Análisis estadístico}

Al final de la colección, se creó una base de datos utilizando el programa Microsoft Excel, versión 2010, y se utilizó la técnica de doble tipeo para identificar y corregir posibles errores durante la transcripción de la información. Los datos se transfirieron al programa spss, versión 22, y luego se analizaron utilizando medidas descriptivas (media, desviación estándar) para la caracterización de la muestra y la frecuencia de las respuestas para el análisis de las preguntas de capacidad aeróbica $(27,28,29,30,32,36$ y 42) contenidas en el cuestionario.

\section{Resultados}

Los resultados descritos en la tabla 3 contienen las frecuencias de respuestas presentadas por los participantes. Con respecto al porcentaje de $\mathrm{vO}_{2 \text { máx }}$ utilizado para desarrollar la capacidad aeróbica, el 2,7\% respondió prescribiendo entre el $21 \%$ y el $40 \%$ del vo $_{2 \text { máx }}$ (alternativa B), el $31.7 \%$ optó por valores entre el $41 \%$ y el $60 \%$ (alternativa C), $40,4 \%$ respondió entre $61 \%$ y $80 \%$ (alternativa D), $2.9 \%$ eligió entre $81 \%$ y $100 \%$ (alternativa E) y $22.4 \%$ de los encuestados eligió no responder (alternativa F). 
Tabla 3. Frecuencia de respuestas a las preguntas relacionadas con el ítem capacidad aeróbica

\begin{tabular}{|c|c|c|c|c|c|c|c|}
\hline \multicolumn{2}{|l|}{$\begin{array}{c}\text { Capacidad } \\
\text { motora }\end{array}$} & \multirow{2}{*}{$\begin{array}{c}\mathrm{VO}_{2} \\
\text { máximo } \\
(\%) \\
-\end{array}$} & \multirow{2}{*}{$\begin{array}{c}\text { FC media } \\
8.7\end{array}$} & \multirow{2}{*}{$\begin{array}{c}\begin{array}{c}\text { Tiempo de } \\
\text { práctica }\end{array} \\
5.8\end{array}$} & \multirow{2}{*}{$\begin{array}{c}\begin{array}{c}\text { Tipo de } \\
\text { ejercicio }\end{array} \\
0.5\end{array}$} & \multirow{2}{*}{$\begin{array}{c}\text { Método } \\
20.7\end{array}$} & \multirow{2}{*}{$\begin{array}{c}\begin{array}{c}\text { Frecuencia } \\
\text { de } \\
\text { evaluación }\end{array} \\
\frac{34.7}{}\end{array}$} \\
\hline & A & & & & & & \\
\hline & B & 2.7 & 42.8 & 27.1 & 75.1 & 25.6 & 11.8 \\
\hline \multirow{4}{*}{$\begin{array}{l}\text { Resistencia } \\
\text { aeróbica }\end{array}$} & C & 31.7 & 26.3 & 40.6 & 4.5 & 11.0 & 30.9 \\
\hline & D & 40.4 & 1.1 & 16.9 & 3.4 & 36.6 & 10.4 \\
\hline & E & 2.9 & 0.2 & 3.1 & 12.4 & 1.8 & 4.7 \\
\hline & $\mathrm{F}$ & 22.4 & 20.9 & 6.7 & 4.1 & 4.3 & 7.6 \\
\hline
\end{tabular}

Fuente: elaboración propia

Con respecto a la frecuencia cardíaca promedio prescrita para desarrollar la capacidad aeróbica, el $8.7 \%$ de los encuestados respondió valores de hasta 140 врм, (alternativa A), $42.8 \%$ optó por valores entre 141 a 160 в м (alternativa B), $26.3 \%$ respondió entre 161 у 180 врм (alternativa C), el $1.1 \%$ optó por valores entre 181 у 200 врм (alternativa D), el $0.2 \%$ respondió valores superiores a 200 в encuestados prefirió no hacerlo (alternativa $\mathrm{F}$ ).

Con respecto al tiempo de actividad aeróbica prescrito para la pérdida de grasa, el $5.8 \%$ de los encuestados respondió entre 0 y 15 minutos (alternativa A), el $27.1 \%$ optó por valores entre 16 y 30 minutos (alternativa B), $40.6 \%$ entre 31 y 45 minutos (alternativa C), el $16.9 \%$ optó por valores entre 46 y 60 minutos (alternativa D), el 3.1\% respondió más de 60 minutos (alternativa E) y el $6.7 \%$ prefirió no hacerlo (alternativa F).

Con respecto al tipo de ejercicio prescrito para desarrollar la capacidad aeróbica, el $0.5 \%$ de los encuestados informó que no prescribió ningún tipo de actividad (alternativa A), el $75.7 \%$ optó por actividades ergométricas, como cinta de correr, bicicleta estática, elíptica, etc. (alternativa B), $4.5 \%$ 
eligió clases de gimnasia como un medio para desarrollar la capacidad aeróbica (alternativa C), $3.4 \%$ utilizó actividades acuáticas (alternativa D), $12.4 \%$ eligió la opción de otras actividades (alternativa E) y el $4.1 \%$ optó por no responder la pregunta (alternativa F).

Con respecto al método utilizado para desarrollar la capacidad aeróbica, el $20.7 \%$ de los encuestados eligió el método continuo (alternativa A), el $25.6 \%$ prefirió el método de intervalo de alta intensidad (alternativa B), el $11 \%$ optó por el método de intervalo de baja intensidad (alternativa C), el $36.6 \%$ respondió el método mixto (alternativa D), el $1.8 \%$ eligió la alternativa E, que correspondía a "otra" y el $4.3 \%$ de los encuestados eligió no responder (alternativa $\mathrm{F}$ ).

Cuando se les preguntó sobre la frecuencia de la aplicación de pruebas de aptitud aeróbica, el $34.7 \%$ de los encuestados informaron que no realizaron pruebas en ningún momento durante el entrenamiento (alternativa A), el $11.8 \%$ reportó que evaluaban a sus estudiantes mensualmente (alternativa B), $30.9 \%$ realizó pruebas trimestrales (alternativa C), $10.4 \%$ semestralmente (alternativa D), 4,7 \% anualmente (alternativa E) y $7.6 \%$ eligió no responder (alternativa $\mathrm{F}$ ).

Además de las preguntas en la tabla 2, se les preguntó a los encuestados sobre la monitorización de la frecuencia cardíaca durante la práctica y el $55.7 \%$ de los encuestados respondieron positivamente a la pregunta, y el resto $(44.3 \%)$ informó que no controlaba la frecuencia cardíaca.

Por lo tanto, se puede afirmar que la mayoría de los profesionales prescriben entre $61 \%$ у $80 \%$ del VO $_{2 \text { máx }}$, adoptan FC entre 141 у 160 врм у usan entre 31 y 45 minutos en el entrenamiento para desarrollar la capacidad aeróbica con objetivos de pérdida de grasa. La mayoría usa la ergometría como un medio de práctica, adopta el método mixto y no realiza pruebas de aptitud aeróbica en ningún momento durante el programa de ejercicio.

Este estudio tuvo como objetivo diagnosticar la metodología utilizada en la práctica por profesionales que trabajan con la prescripción de ejercicio físico en el área de la aptitud física, en relación con el desarrollo de la capacidad aeróbica y sus variables. Como en la literatura se encontraron pocos estudios que abordaran este tema, la discusión de los resultados se basó en la encuesta de datos empíricos y en los supuestos encontrados en la literatura. 
Los resultados descriptivos mostraron que la mayoría de los encuestados $(72.1 \%)$ usaron valores entre $40 \%$ y $80 \%$ del vo $_{2 \text { máx }}$ para desarrollar la capacidad aeróbica. Estos valores son similares al estudio de Branch, Pate y Bourque (2000), en el que se probó el efecto de intensidades moderadas ( $40 \%$ del $\mathrm{vo}_{2 \text { máx }}$ ) en mujeres con una edad promedio de 33 años. En este se descubrió que tal magnitud era suficiente para promover cambios fisiológicos, aunque los resultados no presentaron diferencias significativas en comparación con las intensidades más altas $\left(80 \%\right.$ del $\left.\mathrm{vo}_{2 \text { máx }}\right)$.

Asimismo, los resultados del presente estudio son corroborados por las recomendaciones de Garber y colaboradores (2011) para actividades moderadas, que indican porcentajes entre $40 \%$ y $65 \%$ del $v_{2 \text { máx }}$. Por otro lado, los resultados encontrados para altas intensidades son diferentes de los indi-

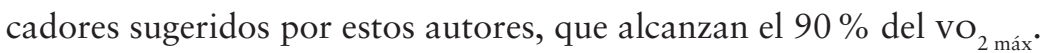

Teniendo en cuenta a los profesionales que respondieron utilizando métodos de alta intensidad para desarrollar la capacidad aeróbica $(25.6 \%)$, solo el $5.26 \%$ de los encuestados indicaron porcentajes de prescripción de $\mathrm{VO}_{2 \text { máx }}$. consistentes con el método sugerido anteriormente (por encima del $80 \%$ ). Gormley y colaboradores (2008) probaron métodos de alta intensidad con porcentajes cercanos al máximo $\left(95 \%\right.$ del $\left.\mathrm{vo}_{2 \text { máx }}\right)$ y encontraron que esta intensidad, en comparación con la intensidad más baja, mostró aproximadamente un $20 \%$ de aumento en la capacidad aeróbica. Los investigadores concluyeron que las intensidades más altas son más eficientes cuando se controla el volumen total de entrenamiento. A pesar de estos resultados, cuando se prescribe el entrenamiento para desarrollar la capacidad aeróbica se deben considerar los hallazgos recientes de Garber y su equipo (2011), que indican una mayor asociación entre las actividades vigorosas y el riesgo de accidente cerebrovascular y muerte por todas las causas.

Con base en estos resultados, se observa que existe una coherencia entre lo que se propone en la literatura y lo que prescriben los profesionales al considerar intensidades moderadas. Sin embargo, cuando se trata de intensidades altas, no parecen considerar las recomendaciones de la literatura y prescriben porcentajes $\mathrm{VO}_{2 \text { máx }}$ por debajo de los valores propuestos.

Además, se debe destacar el alto porcentaje de encuestados que eligieron la alternativa "Prefiero no responder" $(22,4 \%)$. Posiblemente, estos encuestados usan otro indicador fisiológico para prescribir la capacidad 
aeróbica y, por lo tanto, no encontraron ninguna alternativa correspondiente entre las opciones.

$\mathrm{Al}$ analizar los resultados relacionados con la FC promedio prescrita por los profesionales para entrenar la capacidad aeróbica, se verificó que aproximadamente el $50 \%$ de los participantes indicaron una FC promedio de hasta 160 врм, correspondiendo, por lo tanto, a los valores sugeridos por Zakarov y Gomes (2003) (Tabla 1). Según los valores correspondientes al porcentaje de $\mathrm{VO}_{2 \text { máx }}$ indicado por la literatura especializada (OMs, 2010; Garber et al., 2011; Mendes et al., 2011), estos resultados corroboran los descritos por Branch, Pate y Bourque (2000), que indican que las mujeres que practican actividades de intensidad moderada a vigorosa presentaban FC entre 110 у 160 врм durante todas las fases de la prueba. Por el contrario, los resultados encontrados por Foster y colaboradores (2001) revelan valores de FC correspondientes a zonas de porcentaje de $\mathrm{VO}_{2 \text { máx }}$ aeróbico más pequeñas (aproximadamente 85 a 135 вPM) que las encontradas en este estudio.

A pesar de la debilidad señalada por algunos estudios en la literatura con respecto al uso de la FC para la prescripción y el control de ejercicios aeróbicos debido a su gran variabilidad, especialmente en individuos entrenados, se debe enfatizar en que los profesionales en su práctica no consideran estos fracasos en muchas situaciones y eventualmente adoptan zonas de entrenamiento de FC que están disponibles en sitios web de prescripción de ejercicios, manuales de equipos de ejercicios y libros científicos publicados en el área. Los resultados revelaron que existe una correspondencia entre las recomendaciones de la literatura y lo que sucede en la práctica de ejercicios aeróbicos. Sin embargo, se debe destacar el alto porcentaje de participantes $(20.9 \%)$ que optaron por la alternativa "Prefiero no responder", lo que indica que es posible que los profesionales no utilicen la FC como marcador fisiológico o aún no estén seguros de los valores informados para este propósito.

En cuanto a la pregunta sobre la duración de la estadía en minutos de actividad aeróbica para la pérdida de grasa, más de la mitad de los profesionales $(60.6 \%)$ optó por los tiempos correspondientes a las recomendaciones de Donnelly y su equipo de investigadores (2009), que indican valores entre 250 y 300 minutos por semana o más; es decir, aproximadamente 42 minutos por día para la pérdida de peso corporal. Esta duración 
de la estadía ha sido corroborada por McTiernan y colaboradores (2007), quienes informaron pérdidas alrededor de $1.4 \mathrm{~kg}$ en mujeres y $1.8 \mathrm{~kg}$ en hombres después de 12 meses de práctica moderada a vigorosa durante 300 minutos por semana.

Entre los que respondieron de acuerdo con las recomendaciones en la literatura, solo el $33.7 \%$ optó por las intensidades máximas de $\mathrm{vo}_{2}$ (intensidades moderadas), otro $39.2 \%$ sugirió usar intensidades más altas (intensidades superiores al $80 \%$ del $\mathrm{vo}_{2 \text { máx }}$ ) y el resto optó por bajas intensidades.

Estos resultados demuestran que la comprensión de los profesionales de la intensidad de los ejercicios aeróbicos, así como el tiempo dedicado a esta actividad, difiere de las recomendaciones de la comunidad científica, ya que una pequeña porción de profesionales prescribe las intensidades y tiempos correspondientes para la pérdida de grasa.

Otro dato importante encontrado en este estudio se refiere al porcentaje de participantes que eligieron tiempos por debajo de los recomendados en la literatura. Aproximadamente el $32.9 \%$ de los encuestados indicaron prescribir hasta 30 minutos de actividades aeróbicas para la pérdida de grasa y otro $7 \%$ eligieron no responder la pregunta, probablemente porque tenían dudas sobre los valores propuestos o realmente no los conocían.

Berk, Hubert y Fries (2006) no encontraron efectos significativos sobre la pérdida de peso corporal en sujetos que realizaron menos de 60 minutos diarios al menos 5 veces por semana. Teniendo en cuenta sus recomendaciones, solo el $3.1 \%$ de los encuestados optó por momentos similares; y entre estos, el $35.7 \%$ prescribió intensidades moderadas según lo indicado por el autor.

En general, los resultados con respecto al tiempo prescrito por los profesionales para la pérdida de grasa revelan pocas bases teóricas de estos que, al prescribir entrenamiento para este objetivo, optaron por valores similares a los recomendados en la literatura para mantener el peso corporal y mejorar el $\mathrm{VO}_{2 \text { máx }}$, pero que no son adecuados para bajar de peso.

Este desajuste puede ser el responsable del pobre éxito de los programas de pérdida de peso propuestos por profesionales. Esto también puede justificar la baja motivación y la alta tasa de abandono de los practicantes de aeróbicos que asisten a estos programas.

Con respecto al tipo de ejercicio prescrito para desarrollar la capacidad aeróbica, la mayoría absoluta $(75.1 \%)$ indicó la ergometría como un 
medio para desarrollar esta capacidad. La elección entre profesionales para actividades como caminar y andar en bicicleta, simulada por el equipo de ejercicio y descrito como ejemplos en el cuestionario, puede explicarse por el hecho de que estas actividades facilitan el control de las variables de volumen e intensidad relacionadas con la capacidad aeróbica. En este tipo de actividad, el monitoreo del ritmo cardíaco, la velocidad de movimiento y las cargas, a menudo, lo realiza el equipo mismo para ayudar al profesional que, en muchos casos, no está disponible para controlar todo el período de entrenamiento.

Además, corregir una de estas variables para mantener la intensidad del entrenamiento dentro de la zona objetivo establecida puede ser más eficiente que actividades más complejas y variables. La elección de este tipo de ejercicio por parte de los profesionales también puede haber sido motivada por la estructura disponible para la práctica, ya que muchos lugares de entrenamiento no ofrecen espacios o equipos adecuados para actividades acuáticas y gimnásticas, otras opciones contenidas en el cuestionario.

Con respecto al método utilizado para desarrollar la capacidad aeróbica, a pesar de la mayor frecuencia de encuestados que optaron por el método mixto $(36.6 \%)$, otros métodos también presentaron un buen porcentaje de elección entre los participantes de este estudio. Vale la pena mencionar el método de intervalo de alta intensidad, que fue la elección del 25,6 \% de los profesionales, y el método continuo, que presentó una frecuencia de respuesta del $20.7 \%$.

La elección del método mixto de capacitación por parte de la mayoría de los encuestados lleva a creer que estos profesionales saben poco sobre la aplicabilidad de otros métodos, o que a menudo usan más de un método de capacitación para el mismo propósito. Esto los llevó a elegir la alternativa "Mixto", porque representa una combinación de métodos destinados para el mismo fin. Es importante destacar el porcentaje de profesionales que utilizaron el método del intervalo de alta intensidad, lo que indica una tendencia observada en la investigación actual que aborda el efecto del entrenamiento en intervalos de alta intensidad sobre el desarrollo aeróbico.

La pregunta sobre las pruebas para evaluar la aptitud aeróbica indicó que el $34.7 \%$ de los profesionales no realizan pruebas para este propósito. Estos datos no corresponden a las recomendaciones en la literatura, que destacan la importancia del diagnóstico periódico y el control de los niveles 
de aptitud aeróbica de los profesionales, y plantean la cuestión del nivel de compromiso de los profesionales con los problemas de seguridad y salud que involucran al sujeto. En contraste, otros $(30.9 \%)$ de los profesionales indicaron pruebas cada tres meses, como recomiendan los expertos en el campo.

\section{Conclusiones}

La pregunta principal que orientó este estudio se refiere a la comprensión de la metodología utilizada por los profesionales del ejercicio para la prescripción de programas de actividad física. Con base en los resultados descritos, se puede apreciar que, en general, los profesionales usan valores porcentuales de $\mathrm{VO}_{2 \text { máx }}$ correspondientes a los recomendados por expertos en el campo. Sin embargo, esto solo se aplica para intensidades moderadas, siendo divergentes de ellos cuando se consideran intensidades altas. Del mismo modo, se encontró que los valores medios de FC prescritos para desarrollar la capacidad aeróbica y las recomendaciones de la literatura correspondían. Estos resultados indican que la práctica profesional parece estar basada en recomendaciones científicas.

Con respecto al tiempo de práctica para la pérdida de grasa, la mayoría de los encuestados indicaron tiempos correspondientes a los recomendados en la literatura, pero solo un tercio de ellos optó por intensidades adecuadas para este propósito. Por lo tanto, solo una parte de la metodología adoptada por los profesionales para la pérdida de peso corresponde a las recomendaciones encontradas en la literatura.

La elección de ejercicios para entrenar la capacidad aeróbica lleva a creer que los profesionales eligen actividades que facilitan el monitoreo de las variables de volumen e intensidad relacionadas con la capacidad aeróbica. Sin embargo, los profesionales buscan utilizar la estructura disponible para ellos en los diferentes entornos en que actúan.

A pesar de la superioridad del método mixto para desarrollar la capacidad aeróbica, el porcentaje de similitud observado entre las diferentes alternativas revela una comprensión por parte de los profesionales con respecto a las preguntas relacionadas con la elección del método de entrenamiento más apropiado. No obstante, no existe un predominio absoluto 
de un método particular y posiblemente elegirlo depende directamente del conocimiento y las preferencias de práctica de los profesionales.

El alto porcentaje de profesionales que indicaron que no realizan ningún tipo de prueba para evaluar la aptitud aeróbica en ningún momento, revela una situación preocupante que ocurre de manera rutinaria en diferentes lugares de ejercicio físico, y plantea una pregunta sobre el grado de compromiso del profesional con las preguntas. Los problemas de seguridad y salud de los estudiantes dejan dudas sobre la efectividad real de sus programas de ejercicio que no tienen en cuenta los datos de la prueba.

Dados estos hallazgos, se debe resaltar la importancia de aplicar este cuestionario en otras poblaciones, con el fin de conocer la metodología adoptada por los profesionales en diferentes regiones del país y así poder dirigir nuevas investigaciones sobre el tema. Lo anterior con el propósito de ayudar a los profesionales y servir como base de estudios para mejorar la base curricular universitaria.

\section{Referencias}

Almeida, M., Ricardo, D. y Araújo, C. (2005). Variabilidade da frequência cardíaca em um teste de exercício verdadeiramente máximo. Socerj, 18(6), 534-41.

Almeida, M. (2007). Frequência cardíaca e exercício: uma interpretação baseada em evidências. Revista Brasileira de Cineantropometria y Desempenho Humano, 9(2),196-202.

Araújo, C., Herdy, A. y Stein, R. (2013). Medida do Consumo Máximo de Oxigênio: Valioso Marcador Biológico na Saúde e na Doença. Arq. Bras. Cardiol, 100(4), 51-3.

Arts, F. y Kuipers, H. (1994). The Relation Between Power Output, Oxygen Uptake and Heart Rate in Male Athletes. International Journal of Sports Medicine, 15(05), 228-31. DoI: https://doi.org/10.1055/s-2007-1021051

Astrand, P. y Rodahl, K. (1986). Textbook of work physiology. New York: McGraw-Hill.

Berk, D., Hubert, H. y Fries, J. (2006). Associations of changes in exercise level with subsequent disability among seniors: A 16-year longitudinal study. The Journals of Gerontology Series A: Biological Sciences and Medical Sciences, 61(1), 97-102. DoI: https://doi.org/10.1093/gerona/61.1.97 
Blair, S., Kohl, H., Paffenberger, R., Clark, D., Cooper K. y Gibbons, L. (1989) Physical fitness and all-cause mortality: A prospective study of healthy men and women. Jama, 262(17), 2395-401. DoI: https://doi.org/10.1001/ jama.1989.03430170057028

Branch, J., Pate, R. y Bourque, S. (2000). Moderate intensity exercise training improves cardiorespiratory fitness in women. Journal of Women's Health y GenderBased Medicine, 9(1), 65-73. DoI: https://doi.org/10.1089/152460900318984

Caputo, F. y Denadai, B. (2004). Resposta do $\mathrm{VO}_{2}$ e tempo de exaustão durante a corrida realizada na velocidade associada ao $\mathrm{VO}_{2 \max }$ : aplicações para o treinamento aeróbio de alta intensidade. Rev. Bras. Cienc. Esporte, 26(1), 19-31.

Carvalho, J. y Campbell. C. (2012). A frequência cardíaca como fator determinante da intensidade nos exercícios aeróbios realizados no meio líquido. Motricidade. 8(S2), 764-9. Recuperado de https://www.redalyc.org/pdf/2730/Resumenes/ Resumo_273023568091_5.pdf

Casties, J., Mottet, D. y Le Gallais, D. (2006). Non-linear analyses of heart rate variability during heavy exercise and recovery in cyclists. International Journal of Sports Medicine, 27(10), 780-5. DoI: https://doi.org/10.1055/s-2005-872968

Church, T., LaMonte, M., Barlow, C. y Blair, S. (2005). Cardiorespiratory Fitness and Body Mass Index as Predictors of Cardiovascular Disease Mortality Among Men With Diabetes. Archives of Internal Medicine, 165(18), 2114. DOI: 10.1001/archinte.165.18.2114

Cunha, F., Midgley, A., Monteiro, W. y Farinatti, P. (2010). Influence of cardiopulmonary exercise testing protocol and resting $\left.\mathrm{VO}_{2}\right)$ assessment on $\% \mathrm{HR}(\max )$, $\% \mathrm{HRR}, \% \operatorname{VO}\left({ }_{2 \max }\right)$ and $\% \operatorname{VO}\left({ }_{2}\right)$ R relationships. International Journal of Sports Medicine, 31(5), 319-26. DoI: https://doi.org/10.1055/s-0030-1248283

Denadai, B. (1995). Consumo máximo de oxigênio: Fatores determinantes e limitantes. Revista Brasileira de Atividade Física e Saúde, 1(1), 85-94. DoI: https:// doi.org/10.12820/rbafs.v.1n1p85-94

Donnelly, J., Blair, S., Jakicic, J., Manore, M., Rankin, J. y Smith, B. (2009). Appropriate Physical Activity Intervention Strategies for Weight Loss and Prevention of Weight Regain for Adults. Medicine y Science in Sports y Exercise, 41(2),459-71. DoI: https://doi.org/10.1249/MSS.0b013e3181949333

Fletcher, G., Blair, S., Blumenthal, J., Caspersen, C., Chaitman, B., Stephen, E. y Piña, I. (1992). Statement on exercise: Benefits and recommendations for physical activity programs for all Americans. A statement for health professionals by the Committee on Exercise and Cardiac Rehabilitation of the Council on 
Clinical Cardiology, American Heart Association. Circulation, 94(4), 857-62. DOI: https://doi.org/10.1161/01.cir.94.4.857

Foster, C., Florhaug, J.A., Franklin, J., Gottschall, L., Hrovatin, L.A., Parker, S. y Dodge. C. (2001). A new approach to monitoring exercise training. Journal of Strength and Conditioning Research, 15(1), 109-15.

Garber, C., Blissmer, B., Deschenes, M., Franklin, B., Lamonte, M., Lee, I. y Swain, D. (2011). American College of Sports Medicine position stand. Quantity and quality of exercise for developing and maintaining cardiorespiratory, musculoskeletal, and neuromotor fitness in apparently healthy adults: Guidance for prescribing exercise. Medicine and Science in Sports and Exercise, 43(7), 133459. DoI: https://doi.org/10.1249/MSS.0b013e318213fefb

Gilman, M. (1996). The use of heart rate to monitor the intensity of endurance training. Sports Medicine (Auckland, N.Z.), 21(2), 73-79. DoI: https://doi. org/10.2165/00007256-199621020-00001

Gomes, K., Perez. A., Carletti, L. y Marques, A. (2016). Heart rate as an indicator for exercise prescription for normal, overweight, and obese adolescents. Motriz, Rio Claro, 22(2), 27-35. DoI: http://dx.doi.org/10.1590/ S1980-6574201600020004

Gormley, S., Swain, D., High, R., Spina, R, Dowling, E., Kotipalli, U. y Gandrakota, R. (2008). Effect of intensity of aerobic training on $\mathrm{VO}_{2 \max }$. Medicine y Science in Sports y Exercise, 40(7), 1336-43. DoI:0.1249/MSS.0b013e31816c4839

Kavouras, S., Panagiotakos, D., Pitsavos, C., Chrysohoou, C., Anastasiou, C., Lentzas, Y. y Stefanadiz, C. (2007). Physical activity, obesity status, and glycemic control: The ATtica study. Medicine and Science in Sports and Exercise, 39(4), 606-11. DoI: 10.1249/mss. 0b013e31803084eb

Kenney, W., Wilmore, J. y Costill, D.L. (2013). Fisiologia do esporte e do exercício (5. ${ }^{a}$ ed.) São Paulo: Manole.

Leitzmann, M.F., Park,Y., Blair, A., Ballard-Barbash R., Mouw,T., Hollenbeck, A. y Schatzkin, A. (2007). Physical Activity Recommendations and Decreased Risk of Mortality. Archives of Internal Medicine, 167(22), 2453-60. DoI: https:// doi.org/10.1001/archinte.167.22.2453

Lounana, J., Campion, F., Noakes, T. y Medelli, J. (2007). Relationship between $\% \mathrm{HR}_{\max }$, \% HR reserve, $\% \mathrm{VO}_{2}$ max and $\% \mathrm{VO}_{2}$ reserve in elite cyclists. Medicine and Science in Sports and Exercise, 39(2), 350-7. DoI: 10.1249/01. mss.0000246996.63976.5f

Mann,T., Lamberts, R. y Lambert, M. (2013). Methods of prescribing relative exercise intensity: Physiological and practical considerations. Sports 
Medicine (Auckland, N.Z.), 43(7), 613-625. DoI: https://doi.org/10.1007/ s40279-013-0045-x

McTiernan, A., Sorensen, B., Irwin, M., Morgan, A., Yasui,Y., Rudolph, R., Surawicz, C., Lampe, W., Lampe, P., Ayub, K. y Potter, J. (2007). Exercise effect on weight and body fat in men and women. Obesity, 15(6), 1496-512. DOI: https://doi.org/10.1038/oby.2007.178

Mendes, R., Sousa, N. y Barata, J. (2011). Actividade física e saúde pública. Recomendações para a prescrição de exercício. Acta Médica Portuguesa, 24(6), 1025-30. Recuperado de https://pdfs.semanticscholar.org/c901/88ee4de b0df6859064848a586ad8f8bc20ce.pdf

Myers, J., Prakash, M. Froelicher, V., Do, D., Partington, S. y Atwood, J. (2002). Exercise capacity and mortality among men referred for exercise testing. The New England Journal of Medicine, 346(11), 793-801. DoI: https://doi. org/10.1056/nejmoa011858

Panton, L., Graves, J., Pollock, M., Garzarella, L., Carroll, J., Leggett, S., Guillen, Lowenthal, D. y Guillen, G. (1996). Relative heart rate, heart rate reserve, and $\mathrm{vO}^{2}$ during submaximal exercise in the elderly. Journals of Gerontology - Series A Biological Sciences and Medical Sciences, 51(4),165-171. DoI: https://doi. org/10.1249/00005768-199205001-01111

Raxwal, V., Shetler, K, Morise, A., Do, D., Myers, J., Atwood, J. y Froelicher, V. (2001). Simple Treadmill Score To Diagnose Coronary Disease. Chest, 11(6), 1933-40. DOI: https://doi.org/10.1378/chest.119.6.1933

Rosenberger, F., Meyer, T., Gäßler, N., Faude, O. y Kindermann. W. (2010). Exercise at given percentages of $\mathrm{VO} 2 \mathrm{max}$ : Heterogeneous metabolic responses between individuals. Journal of Science and Medicine in Sport, 13, 74-9. DOI: https://doi.org/10.1016/j.jsams.2008.12.626

Seiler, K. y Kjerland, G. (2006). Quantifying training intensity distribution in elite endurance athletes: is there evidence for an "optimal" distribution? Scandinavian Journal of Medicine \& Science in Sports, 16(1), 49-56. DOI: https://doi.org/10.1111/j.1600-0838.2004.00418.x

Silva, C., Franklin, B., Forman, D. y Araújo, C. (2016). Influence of age in estimating maximal oxygen uptake. Journal of Geriatric Cardiology: JGC, 13(2), 12631. DOI: https://doi.org/10.11909/j.issn.1671-5411.2016.02.010

Skinner, J., Gaskill, S., Rankinen,T., Leon, A., Rao, D., Wilmore, J. y Bouchard, C. (2003). Heart rate versus $\% \mathrm{VO}_{2 \max }$ : Age, sex, race, initial fitness, and training response - Heritage. Medicine and Science in Sports and Exercise, 35(11), 1908-13. DoI: https://doi.org/10.1249/01.MSS.0000093607.57995.E3 
Sui, X., LaMonte, M., Laditka, J., Hardin, J., Chase, N., Hooker, S. y Blair, S. (2007). Cardiorespiratory Fitness and Adiposity as Mortality Predictors in Older Adults. Jama, 298(21), 2507. Dor: https://doi.org/10.1001/jama.298.21.2507

Swain, D. y Franklin, B. (2002). $\mathrm{vo}_{2}$ reserve and the minimal intensity for improving cardiorespiratory fitness. Medicine and Science in Sports and Exercise, 34(1), 152-7. DoI: https://doi.org/10.1097/00005768-200201000-00023

Swain, D. y Leutholtz, B. (1997). Heart rate reserve is equivalente to $\% \mathrm{vo}_{2}$ reserve, not to $\% \mathrm{vO}_{2 \max }$. Medicine and Science in Sports and Exercise, 29(3), 410-4. DOI: https://doi.org/10.1097/00005768-199703000-00018

Swain, D., Leutholtz, B., King, M., Haas, L. y Branch, J. (1998). Relationship between $\%$ heart rate reserve and $\% \mathrm{VO}_{2}$ reserve in treadmill exercise. Medicine and Science in Sports and Exercise, 30(2), 318-21. DoI: https://doi. org/10.1097/00005768-199802000-00022

Verenguer, R. (2003). Mercado de trabalho em educação física: Significado da intervenção profissional à luz das relações de trabalho e da construção da carreira (tesis inédita de doctorado). Universidade Estadual de Campinas, Campinas, Brasil.

Warren, T.Y., Barry, V., Hooker, S.P., Sui, X., Church, T.S. y Blair, S.N. (2010). Sedentary behaviors increase risk of cardiovascular disease mortality in men. Medicine \& Science in Sports \& Exercise, 42(5), 879-85. DoI: https://doi. org/10.1249/mss.0b013e3181c3aa7e

Weltman, A., Weltman, J., Rutt, R., Seip, R., Levine, S., Snead, D, Kaiser, D. y Rogol, A. (1989). Percentages of maximal heart rate, heart rate reserve, and $\mathrm{VO}_{2}$ peak for endurance training intensity in sedentary women. International Journal of Sports Medicine, 10(3), 212-6. DOI: 10.1055/s-2007-1024903

Zakharov, A. A y Gomes, A.C. (2003). Ciência do treinamento desportivo (2. ${ }^{\mathrm{a}} \mathrm{ed}$.) Río de Janeiro: Grupo Palestra Sport. 